\title{
Abnormal vertical optokinetic nystagmus in infants and children
}

\author{
Siobhan Garbutt, Christopher M Harris
}

\begin{abstract}
Aims-To determine if testing vertical optokinetic nystagmus (VOKN) has a role in the clinical assessment of infants and children.

Methods-A large field projection system
\end{abstract} was developed with which optokinetic nystagmus (OKN) could be stimulated in any direction. Gross abnormalities in the response were detected simply by observation.

Results-VOKN was tested in 144 children using this $O K N$ projection system. 26 of these children had abnormal VOKN; 13 had a vertical saccade initiation failure "ocular motor apraxia" (in either direction, up/down, or in both) and $13 \mathrm{had}$ absent VOKN (in either direction, up/ down, or in both). Nine of the children with an up and/or down vertical saccade initiation failure (VSIF) had a neurometabolic disease (two had Niemann-Pick disease type C, five had Gaucher disease type III, one had Gaucher disease type II, and one had Gaucher disease type I). Five children with a VSIF had an abnormality identified by a magnetic resonance imaging (MRI) scan of the brain. In two of these children there was a focal lesion of the rostral midbrain. In 11 of the children with absent up and/or down VOKN an MRI scan revealed an abnormality. This involved the brainstem and/or the cerebellum in 10. Absent up and/or down VOKN was found in association with Joubert syndrome, Leigh disease, and cerebral palsy. Conclusion-VOKN testing has a useful role in detecting neurological abnormalities in infants and children. Detection of abnormal VOKN should indicate further investigations for a neurometabolic disease or an abnormality involving the cortex, brainstem, and/or cerebellum. Abnormal VOKN but normal horizontal $\mathrm{OKN}$ is highly suggestive of a rostral midbrain lesion.

(Br F Ophthalmol 2000;84:451-455)

Optokinetic nystagmus (OKN) is a reflexive oscillation of the eyes induced by motion of the whole or, at least, a very large part of the visual field. The OKN response consists of an alternating sequence of following movements (slow phases) and fast resetting saccades (quick phases). OKN can be elicited even from the newborn ${ }^{12}$ and if the whole visual field moves it is virtually impossible to suppress.

Horizontal OKN (HOKN) has been studied extensively. Among its many roles in the clinical assessment of infants and children, testing $\mathrm{HOKN}$ is useful in identifying lesions that affect the horizontal optokinetic pathway (involving the cortex, brainstem, or cerebellum). A unilateral lesion of this pathway will result in poorer $\mathrm{HOKN}$ to the same side as the lesion. ${ }^{3} \mathrm{~A}$ complete absence of $\mathrm{HOKN}$ can indicate a bilateral lesion affecting the horizontal optokinetic neural pathway. HOKN testing is also useful in the identification of horizontal saccade initiation failure (HSIF) or "ocular motor apraxia". Traditionally, HSIF has been identified by recognition of head thrusting and/or synkinetic blinking. However, as these compensatory behaviours are not always adopted, identification of absent HOKN quick phases is considered to be a more reliable test for $\mathrm{HSIF}^{4}$

The neural pathways controlling vertical OKN (VOKN) have not been clearly established, therefore abnormalities in the vertical optokinetic response are not as well understood as abnormal HOKN. However, if we consider the quick phase of VOKN to be a vertical saccade and the slow phase to be a vertical smooth pursuit movement, we can go some way towards explaining the neural control of VOKN. The frontal eye fields and superior colliculi are responsible for the generation of vertical (and horizontal) saccades. ${ }^{5}$ From here the pathways controlling vertical saccades have not been fully elucidated, but it is believed that before reaching the vertical ocular motor nuclei they are relayed via the rostral interstitial nuclei of the medial longitudinal fasciculus (riMLFs). This pair of nuclei in the rostral midbrain contain vertical saccade burst neurons, ${ }^{6-8}$ and thus are crucial for the generation of vertical saccades. The pathways responsible for vertical smooth pursuit most likely originate in the posterior hemisphere. ${ }^{5}$ Again the pathways to the vertical ocular motor nuclei are not fully understood. In the monkey, at least, the dorsolateral pontine nuclei (DLPN) are considered to be a crucial relay since following unilateral DLPN lesions vertical and horizontal smooth pursuit are
Accepted for publication 8 December 1999 
impaired. ${ }^{9}$ It is thought that the flocculus of the cerebellum is likely to be involved since bilateral flocculectomy results in major abnormalities in the slow phases of VOKN and HOKN. ${ }^{10}$ Additionally, it is known that neurons that discharge in relation to vertical pursuit can be found in the dentate nucleus and in the Y-group of the vestibular nuclei, ${ }^{11}{ }^{12}$ and some vertical pursuit signals are also found in the medial longitudinal fasciculus (MLF)..$^{13}$ The final supranuclear relay in the midbrain reticular formation has not been identified, but the interstitial nucleus of Cajal is considered to play an important part in the control of vertical smooth pursuit..$^{15}$ Thus, we can infer that the neural pathways controlling VOKN most likely involve the cortex, brainstem (particularly the rostral midbrain), and cerebellum, and presumably abnormalities of these areas could result in abnormal VOKN.

HOKN may be effectively elicited by rotating a patterned curtain around a stationary subject. VOKN, on the other hand, is more difficult to stimulate and record effectively, and this is probably the reason why it has not been as thoroughly investigated as HOKN. To determine if VOKN has a useful role in the clinical assessment of infants and children we developed a projection system with which we could stimulate $\mathrm{OKN}$ in any direction.

\section{Methods}

OKN was elicited by back projecting a black and white grating on to a large viewing screen. This screen was $165 \mathrm{~cm}$ wide and $120 \mathrm{~cm}$ high. The children sat in a hydraulic chair facing the screen, their eyes level with its centre, at a distance of $60 \mathrm{~cm}$ (Fig 1). Older children sat alone, younger children sat on a parent's lap. The head was immobilised by a headrest or was held by the parent or the examiner.

Horizontal black and white stripes moved vertically to elicit VOKN and vertical stripes moved horizontally to elicit HOKN. The stimulus covered approximately 108 degrees of the horizontal visual field and 90 degrees of the vertical visual field. The grating had a spatial frequency of 0.04 cycles/degree and moved at a velocity of 28 degrees/second. Lighting was at a low ambient level.

Horizontal and vertical OKN were tested in each child and OKN was recorded as present,

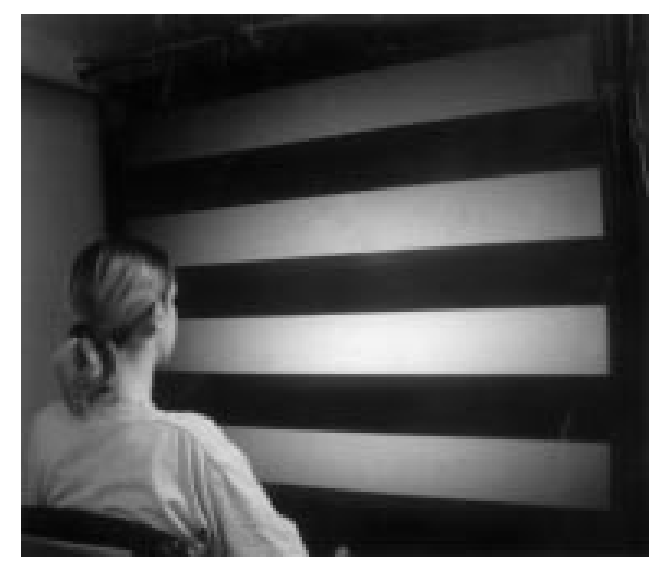

Figure 1 View of the OKN projection screen. absent, or lock up. Lock up identified cases of saccade initiation failure (SIF). In SIF the quick phases of OKN are intermittently missed and so the unchecked slow phase drives the eyes to the mechanical limit of gaze where they remain "locked up" until a quick phase eventually occurs.

As part of a standard eye movement protocol, VOKN was tested in 144 children referred to the eye movement laboratory at Great Ormond Street Hospital. These children were referred from ophthalmology, metabolic, and neurology departments over a period of 1 year. Reasons for referral included unusual eye movements, unusual head movements, visual unresponsiveness, or suspicion of a neurometabolic disease.

\section{Results}

In 118 of the 144 children tested, a response to an upward and a downward moving optokinetic stimulus was observed with no evidence of lock up. These children were considered to have "normal" VOKN, although their eye movements were not recorded objectively and therefore any subtle differences in the response elicited by an upward moving stimulus compared with that elicited by a downward moving stimulus would not have been detected. The remaining 26 children had obviously abnormal VOKN. Of these, 13 had a vertical SIF (VSIF) (in either direction, up/down, or in both) and 13 had absent VOKN (in either direction, up/down, or in both). There were 12 males and 14 females.

Nine of the children with a VSIF had a neurometabolic disease (two had Niemann-Pick disease type C, five had Gaucher disease type III, one had Gaucher disease type II, and one had Gaucher disease type I). Five children with a VSIF had an abnormality identified by a magnetic resonance imaging (MRI) scan of the brain.

In 11 of the children with absent up and/or down VOKN an MRI scan revealed an abnormality. This involved the brainstem and/or the cerebellum in 10 . These results are summarised in Table 1.

All 26 of the children with abnormal VOKN had vertical and horizontal ocular movements on the doll's head manoeuvre. In all the children with a VSIF vertical smooth pursuit appeared normal, and saccades in the direction opposite to lock up could only be elicited by the use of a head thrust or by blinking synkinetically, if at all. In those with a complete absence of OKN, no smooth pursuit could be elicited in any direction. In those with an absence of up VOKN, smooth pursuit was absent upwards but present downwards. The reverse was true for those with absent down VOKN. Vertical saccades were not formally tested because we have found these very difficult to elicit reliably even in normal children. All children had pupils that were equal and reactive to light apart from one child (patient 1) who had a dilated right pupil that did not react to light. 


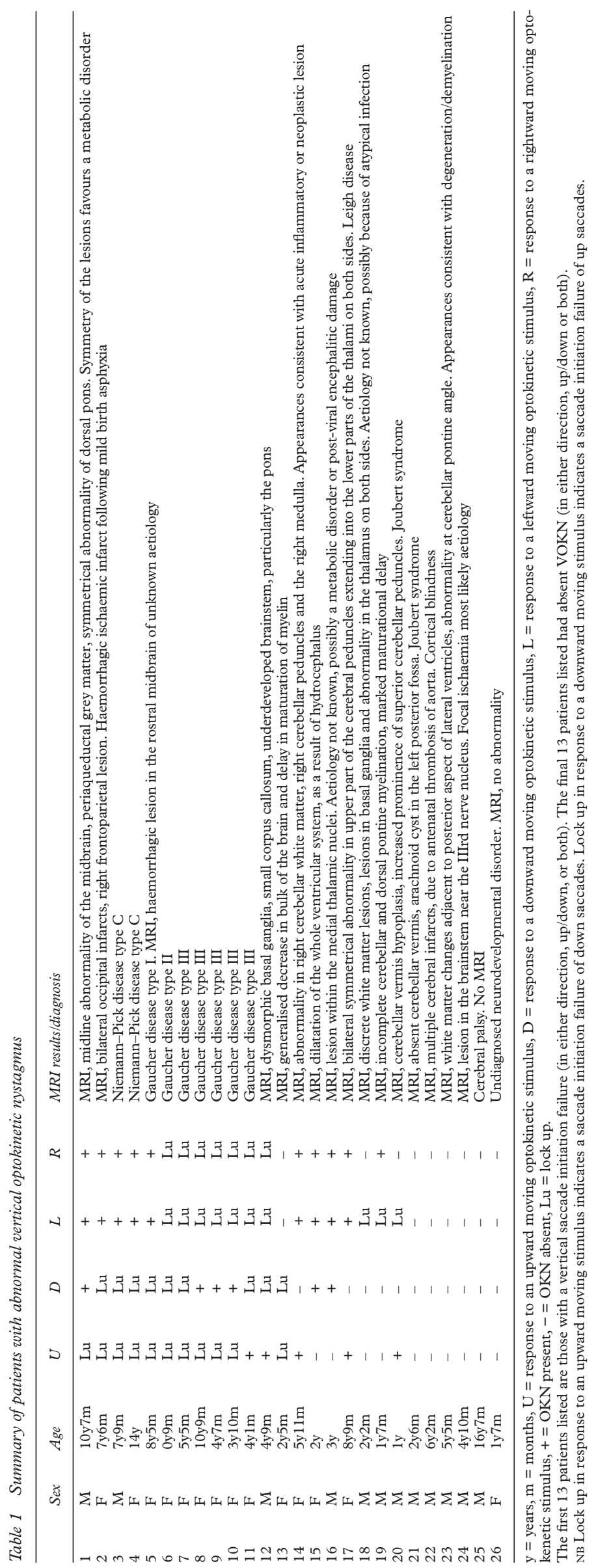

\section{Discussion}

We first consider those children with a VSIFthat is, absence of upward and/or downward quick phases and thus lock up on VOKN testing, and discuss the most relevant cases. As mentioned above, the riMLFs, part of the midbrain reticular formation, are believed to be crucial for the generation of vertical saccades and presumably optokinetic quick phases. Experimental lesions and clinicopathological studies suggest that from the midbrain reticular formation, the efferent fibres for vertical saccades/optokinetic quick phases and those for vertical smooth pursuit travel together to the vertical oculomotor nuclei. ${ }^{76}$ All the children described in this study with a VSIF had normal vertical smooth pursuit, therefore, their VSIF is most likely to be the result of a lesion of the riMLFs themselves or afferent fibres to them. This is consistent with an abnormality of the midbrain, periaqueductal grey matter, and dorsal pons identified in one child (patient 1) and with an underdevelopment of the brainstem, particularly the pons, in another (patient 12).

One child (patient 2) was found to have a lesion involving the right frontoparietal region. This could account for the VSIF as it may have affected the frontal eye fields. However, since the frontal eye fields, along with the superior colliculi, are responsible for generating both vertical and horizontal saccades, ${ }^{5}$ we would expect a HSIF to also be evident. This was not the case. It is more likely, therefore, that there was a lesion in the area of the rostral midbrain. Unfortunately, neuroimaging of this area was inconclusive.

Acquired VSIF has been documented in certain neurometabolic diseases, such as NiemannPick disease type C, Gaucher disease type II and III, Tay-Sachs disease and Wilson disease. ${ }^{17-23}$ SIF in neurometabolic disease indicates dysfunction of supranuclear ocular motor control. Nine of the children identified with VSIF in this study had a neurometabolic disease. This relatively large number of children with a neurometabolic disease and a VSIF reflected the pattern of patients referred to our laboratory. In this study, two children (patients 3 and 4) with a VSIF were diagnosed as having Niemann-Pick disease type C. Deficits in the generation of rapid vertical eye movements are frequently seen in cases of Niemann-Pick type $\mathrm{C}^{17}{ }^{18}$ and indeed a VSIF may be the presenting sign of the disease, ${ }^{23}$ as was the case with one child (patient 4) reported here. Neville and co-workers ${ }^{18}$ described the typical sequence of ocular motor abnormalities seen in Niemann-Pick disease. This begins with loss of vertical saccades and absence of $\mathrm{OKN}$ quick phases (particularly affecting down gaze), followed by impairment of vertical smooth pursuit and then similar involvement of horizontal movements with reduced velocity of saccades to voluntary and optokinetic stimuli. Finally convergence is affected. The two patients reported in this study were presumably in the early stages of disease, since only their vertical saccades and optokinetic quick phases (both up and down) were affected. Vertical 
smooth pursuit, horizontal saccades, HOKN, and convergence were all intact.

The ocular motor abnormalities in neuronopathic Gaucher disease have been outlined recently ${ }^{24}$ and these include HSIF, strabismus, slow horizontal and downward saccades, and an abnormal vestibulo-ocular reflex. HSIF is the most consistent finding and is frequently the first sign of neurological involvement. Thus, identification of this in patients with Gaucher disease is crucial for their diagnosis and management as it indicates that they have the neuronopathic form of the disease- that is, type II or III. In our experience, when there is dysfunction of supranuclear ocular motor control in Gaucher disease, horizontal gaze is always affected first and thereafter involvement of vertical eye movements may indicate progression of the disease. Therefore we were surprised to find that in one child (patient 5) there was only an SIF in the vertical plane, with normal HOKN. However, an MRI scan revealed a lesion in the rostral midbrain with the appearance of an old haemorrhage. This presumably was the cause of the VSIF. Thus, despite the identification of an SIF her current diagnosis is of Gaucher disease type I (that is, the non-neuronopathic form), although she is still under investigation.

Of the 13 cases of VSIF identified in this study, seven had an HSIF. HSIF has been associated with a wide range of condition $\mathrm{s}^{25}$ but is frequently found to be idiopathic. Of the 13 cases of VSIF identified in this study none was idiopathic. Thus, the presence of a VSIF, whether associated with a HSIF or not, should always indicate investigations for a more sinister cause. Equally, identification of HSIF should lead to testing of the vertical plane.

A VSIF will not necessarily involve both upward and downward saccades and as we have seen here saccades in only one direction may be affected. It has been suggested that saccades may be lost in only one vertical direction because burst neurons for upward and downward saccades are topographically arranged within the riMLFs. Büttner-Ennever and colleagues ${ }^{7}$ suggested that the medial aspect of the riMLFs contain neurons involved in upgaze while neurons from the lateral portion are primarily involved in downgaze. Thus, it is plausible that a more medially situated lesion would result in a VSIF affecting only upward saccades and a laterally placed lesion a VSIF of downward saccades, while a more extensive lesion would lead to a complete VSIF. This view is controversial, however, and others $^{16}$ have reached opposite conclusions regarding the arrangement of vertical burst neurons within the riMLFs. In neurometabolic diseases it appears that downward saccades are often affected before upward saccades. This has been observed in Niemann-Pick disease ${ }^{18}$ and in Gaucher disease. ${ }^{24}$ This preferential loss of downward saccades was also evident in the children with Gaucher disease type III in this study.

Next we consider those 13 children with absent VOKN-that is, those with no response to an upward and/or downward moving optokinetic stimulus. As discussed above the VOKN neural pathways most likely involve the cortex, brainstem, and cerebellum. In one child (patient 22) with a complete absence of VOKN there was cortical involvement. In six of the children with absent up and/or down VOKN an abnormality that specifically involved the cerebellum and/or brainstem was identified by MRI scan. One child (patient 14) was reported to have an abnormality of the right medulla and cerebellum, a second (patient 19) had incomplete cerebellar and dorsal pontine myelination, and a third (patient 23) had an abnormality at the cerebellar pontine angle. A lesion in the brainstem near the third nerve nucleus was identified in one child (patient 24). The third nerve nucleus lies partly within the MLF and thus complete absence of $\mathrm{OKN}$, along with an internuclear ophthalmoplegia and a partial third nerve palsy, are consistent findings. Two cases (patients 20 and 21) with abnormal VOKN had a diagnosis of Joubert syndrome. Ocular motor abnormalities have previously been reported in association with Joubert syndrome, ${ }^{26}{ }^{27}$ and it is believed that they are secondary to dysfunction of both the cerebellum and the brainstem. ${ }^{27}$

Four children with absent up and/or down VOKN had a lesion that most probably involved the rostral midbrain. The first child (patient 15) had hydrocephalus and dilatation of the whole ventricular system; dilatation of the third ventricle could affect the rostral midbrain. Three children (patients 16,17, and 18) had lesions that involved the thalamus. Although there have been reports of thalamic lesions affecting vertical gaze $\mathrm{e}^{28-31}$ there is no anatomical documentation for thalamic control of vertical gaze. Therefore, it is more probable in these three cases that there was a disruption of the pathways controlling vertical gaze in the adjacent rostral midbrain.

In this study nine children (patient numbers $1,2,3,4,5,14,15,16$, and 17) had abnormal VOKN but normal HOKN. Of these nine children, six (patient numbers 1, 2, 5, 15, 16, and 17) had a lesion that most probably specifically involved the rostral midbrain. Thus, it appears that abnormal VOKN but normal HOKN is highly suggestive of a rostral midbrain lesion.

Since we found that VOKN may only be absent in one direction, there clearly is some segregation between the neural pathways for up VOKN and those for down VOKN. However, it is not known to what extent these pathways are separate. Although the VOKN pathways have not been fully elucidated it has been proposed ${ }^{16}$ that the tracts involved in upgaze decussate through the posterior commissure, while those for downgaze do not pass through the commissure and possibly decussate near the oculomotor nuclei. However, from the results of MRI scans alone it is not possible for us to reach any conclusions regarding the exact organisation of these pathways. 


\section{Conclusion}

VOKN testing has a useful role in detecting neurological abnormalities in infants and children. The OKN projection system is an effective clinical tool for testing VOKN. By simple observation, lock up, and thus cases of VSIF, can be identified as can absent VOKN. However, further investigations are required to determine if objective recording of eye movements during VOKN testing would have additional value.

In this study, VSIF was frequently associated with a neurometabolic disease. VSIF was also associated with lesions involving the brainstem, patricularly the rostral midbrain. Absent VOKN was associated with lesions of the cortex, brainstem and/or cerebellum. Abnormal VOKN but normal HOKN is highly suggestive of a rostral midbrain lesion.

We wish to thank the charities Iris Fund and Help a Child to See for their support. We also thank Sarah Benton, Lucinda Carr, Peter Clayton, Helen Cross, Carlos De Sousa, Fenella Kirkham, Jane Leitch, Ken Nischal, Isabelle Russell-Eggitt, Robert Surtees, David Taylor, and Ashok Vellodi for referral of the patients.

1 Gorman JJ, Cogan DG, Gellis SS. An apparatus for grading the visual acuity of infants on the basis of optokinetic nystagmus. Pediatrics 1957;19:1088-92.

2 Dayton GOJ, Jones MH, Aiu P, et al. Developmental study of co-ordinated eye movements in the human infant. Arch Ophthalmol 1964;71:865-70.

3 Baloh RW, Honrubia V, Sills A. Eye tracking and optokinetic nystagmus. Results of quantitative testing in patients with well-defined nervous system lesions. Ann Otol Rhinol Laryngol 1977;86:108-14.

4 Harris CM, Shawkat F, Russell-Eggitt I, et al. Intermittent horizontal saccade failure ('ocular motor apraxia') in children. Br f Ophthalmol 1996;80:151-8.

5 Miller NR. The neural control of eye movements. In: Miller NR, ed. Walsh and Hoyt's clinical neuro-ophthalmology. 4th NR, ed. Walsh and Hoyt's clinical neuro-ophthalmology.
ed. Baltimore: Williams and Wilkins, 1985:608-33.

6 Büttner-Ennever JA. Organization of reticular projections Büttner-Ennever JA. Organization of reticular projections
onto oculomotor neurons. Prog Brain Res 1979;50:619-30. 7 Büttner-Ennever JA, Büttner U, Cohen B, et al. . Vertical gaze paralysis and the rostral interstitial nucleus of the medial longitudinal fasciculus. Brain 1982;105:125-49.

8 Leigh RJ, Zee DS. The neurology of eye movements. 2nd ed. Philadelphia: FA Davis, 1991

9 May JG, Keller EL, Suzuki DA. Smooth-pursuit eye movement deficits with chemical lesions in the dorsolateral pontine nucleus of the monkey. 7 Neurophysiol 1988;59:95277.

10 Waespe W, Cohen B, Raphan T. Role of the flocculus and paraflocculus in optokinetic nystagmus and visualvestibular interactions: effects of lesions. Exp Brain Res 1983;50:9-33.

11 Chubb MC, Fuchs AF. The role of the dentate nucleus and y-group in the generation of vertical smooth eye movements. Ann NY Acad Sci 1981;374:446-54.
12 Chubb MC, Fuchs AF. Contribution of y group of vestibular nuclei and dentate nucleus of cerebellum to generation of vertical smooth eye movements. $\mathcal{F}$ Neurophysiol 1982;48: $75-99$.

13 King WM, Lisberger SG, Fuchs AF. Responses of fibers in medial longitudinal fasciculus (MLF) of alert monkeys during horizontal and vertical conjugate eye movements evoked by vestibular or visual stimuli. F Neurophysiol 1976; 39:1135-49.

14 Pola J, Robinson DA. Oculomotor signals in medial longitudinal fasciculus of the monkey. $\mathcal{f}$ Neurophysiol 1978;41: 245-59.

15 Pierrot-Deseilligny CH, Rivaud S, Samson Y, et al. Some instructive cases concerning the circuitry of ocular smooth pursuit in the brainstem. Neuro-Ophthalmology 1989;9:3142.

16 Pierrot-Deseilligny CH, Chain F, Gray F, et al. Parinaud's syndrome: electro-oculographic and anatomical analysis of six vascular cases with deductions about vertical gaze organization in the premotor structures. Brain 1982;105: 667-96.

17 Sanders MD, Wybar KC. Vertical supranuclear ophthalmoplegia with compensatory head movement. Report of a case plegia with compensatory head movement. Report of a case Strabismi Studio Deditum Congress. London: Kimpton, Strabismi Stud

18 Neville BGR, Lake BD, Stephens R, et al. A neurovisceral storage disease with vertical supranuclear ophthalmoplegia, and its relationship to Niemann-Pick disease. A report of nine patients. Brain 1973;96:97-120.

19 Cogan DG, Chu FC, Reingold D, et al. Ocular motor signs in some metabolic diseases. Arch Ophthalmol 1981;99: 1802-8.

20 Miller NR. Topical diagnosis of neuropathic ocular motility disorders. In: Miller NR, ed. Walsh and Hoyt's clinical neuroophthalmology. 4th ed. Baltimore: Williams and Wilkins, 1985:634-784.

21 Grafe M, Thomas C, Schneider J, et al. Infantile Gaucher's disease: a case with neuronal storage. Ann Neurol 1988;23: 300-3.

22 Vivian AJ, Harris CM, Kriss A, et al. Oculomotor signs in infantile Gaucher disease. Neuro-Ophthalmology 1993;13: $151-5$.

23 Shawkat F, Carr L, West P, et al. Vertical saccade palsy: a presenting sign in Niemann-Pick type IIS. Eur f N Neurol 1994:1:93-5.

24 Harris CM, Taylor DSI, Vellodi A. Ocular motor abnormalities in Gaucher disease. Neuropediatrics (in press).

25 Harris C. Other eye movement disorders. In: Taylor D, ed. Paediatric ophthalmology. 2nd ed. Oxford: Blackwell Science, 1997:897-924.

26 Moore AT, Taylor DSI. A syndrome of congenital retinal dystrophy and saccade palsy - a subset of Leber's amaurosis. Br f Ophthalmol 1984;68:421-31.

27 Lambert SR, Kriss A, Gresty $M$ et al. Joubert syndrome. Arch Ophthalmol 1989;107:709-13.

28 Kobari M, Ishihara N, Yunoki K. Bilateral thalamic infarction associated with selective downward gaze paralysis. Eur Neurol 1987;26:246-51.

29 Fensore C, Lazzarino LG, Nappo A, et al. Language and memory disturbances from mesencephalothalmic infarcts. A clinical and computed tomography study. Eur Neurol 1988;28:51-6.

30 Lazzarino LG, Nicolai A. Aphonia as the only speech disturbance from bilateral paramedian thalamic infarction. Clin Neurol Neurosurg 1988;90:265-7.

31 Ghidoni E, Pattacini F, Galimberti D, et al. Lacunar thalamic infarcts and amnesia. Eur Neurol 1989;29:13-5. 